\title{
Improved Multidimensional Representation Using Shearlets
}

\author{
Dewei Gong ${ }^{1}$, Jianhai Chen ${ }^{1}$, Jinliang Chai ${ }^{1}$, Jizheng $\mathrm{Di}^{1}$
}

Abstract. Despite effective tools in signal and image processing, traditional wavelets methods lose the ability to process multi-dimensional data. In this paper, we describe a new class of multidimensional representation systems, called shearlets. Similar with wavelets, this new representation can be associated with multiresolution analysis. The shearlet representation provides a more effective tool for the geometric representation of multidimensional data. We introduce the affine systems with composite dilations for the shearlet construction and give several examples of shearlets.

Keywords: wavelets, shearlets, affine systems, multidimensional representation

\section{Introduction}

Wavelet representation is thought to be one of the most effective tools in image and signal processing. One of the most useful features of wavelets is that they are efficient to approximate signals containing point-wise singularities[3]. Traditional wavelet methods, however, fail to process multi-dimensional data for their invalidation about the singularities of surfaces [5,8].

In order to overcome the limitation of traditional wavelets, several new image representations have been proposed such as Ridgelets[9], Curvelets[8], Contourlets[10] and Shearlets[11]. The most successful representations include the curvelets introduced by Candes and Donoho. The curvelets are effective for their optimal approximation for two-dimensional piecewise smooth functions. However, they lose the ability to provide a multi-resolution representation of the geometry that traditional wavelets possess. Therefore, it is very challenging to make the discrete implementation of curvelets.

Recently, a new representation called shearlet has been introduced[4-7]. This new representation is very effective in the geometric representation of multidimensional data. Since the shearlet representation possesses a more rigorous mathematical framework, it is easier for the implementation of shearlets[3]. An

\footnotetext{
${ }^{1}$ Jizheng Di (gongdewei123@gmail.com)

Department of Applied Mathematics, Zhejiang University of Technology, 310023 Hangzhou, China

e-mail: gongdewei123@gmail.com
} 
effective construction of shearlets, however, is still a challenging problem for us to tackle.

The paper is organized as follows. In Section 2 we introduce the mathematical theory of shearlet frames. In Section 3 we describe the framework of the affine systems for the construction of shearlets and provide several examples of shearlets. Concluding remarks are drawn in Section 4.

\section{Shearlet Framework}

As described in the introduction, Shearlet representation has many advantages such as directional sensitivity and optimal approximation. Some methods to construct shearlets have been demonstrated in some literatures. Inspired by their work, we propose a new method to construct shearlets by obtaining their Fourier forms on some necessary conditions.

Before constructing usable shearlets, we give the following theorem which makes it theoretically possible to construct certain shealets.

\section{Theorem 2.1}

Let $C$ be an $n \times n$ invertible matrix and $\Delta \subset Z \times Z^{n-1}$ be an index set. Suppose that $\hat{\psi} \in L^{\infty}\left(R^{n}\right)$, where supp $\hat{\psi} \subset \Gamma=\left(-\frac{1}{2}, \frac{1}{2}\right]^{\mathrm{n}} \mathrm{C}^{-1}$ and

$$
\sum_{(j, l) \in \Delta}\left|\hat{\psi}\left(\omega A^{j} B(l)\right)\right|^{2}=|C| \text { (a.e) }
$$

then $\left\{\psi_{j l k}\right\}_{(j, l) \in \Delta, k \in Z^{n}}$ is a Parseval frame for $L^{2}\left(R^{n}\right)$,

where $\psi_{j l k}(\cdot)=|A|^{\frac{1}{2}} \psi\left(B(l) A^{j} \cdot-C k\right)$. Thus, we have the following reconstruction formula

$$
f=\sum_{(j, l) \in \Delta} \sum_{k \in Z^{n}}<f, \psi_{j l k}>\psi_{j l k} \quad \text { for all } f \in L^{2}\left(R^{n}\right)
$$

\section{Construction of Shearlets}

To construct usable shearlets, we need some ancillary functions with special properties. Some ideas of these constructions are adapted from [1] and [2]. The results will be used later.

We start by defining an ancillary function $\theta: \square \rightarrow \square$ as 


$$
\theta(x)= \begin{cases}0 & \text { for } x<0 \\ \sin \frac{2^{n-1} \pi}{3}\left(x-\frac{1}{2}\right)^{n}+\frac{1}{2} & \text { for } 0 \leq x \leq 1 \\ 1 & \text { for } x>1\end{cases}
$$

It is easy to verify that $\theta$ is symmetrical around $\left(\frac{1}{2}, \frac{1}{2}\right)$, and monotonically increasing on [0,1]. A plot of $\theta$ where $n=3$ is shown in Fig. 1(a).

Next, define a smooth bell function $b: \square \rightarrow \square$ as

$$
b(\omega)= \begin{cases}\sin \left(\frac{\pi}{2} \theta(8|\omega|-1)\right) & \text { for } \frac{1}{8} \leq|\omega| \leq \frac{1}{4} \\ \cos \left(\frac{\pi}{2} \theta(4|\omega|-1)\right) & \text { for } \frac{1}{4}<|\omega| \leq \frac{1}{2} \\ 0 & \text { otherwise }\end{cases}
$$

We can easily verify that $b$ is a non-negative real valued function axially symmetric to the $\mathrm{y}$-axis and $b\left( \pm \frac{1}{4}\right)=1$. We further have $\operatorname{supp} b=\left[-\frac{1}{2},-\frac{1}{8}\right] \bigcup\left[\frac{1}{8}, \frac{1}{2}\right]$. A plot of $b$ is shown in Fig. 1(b).

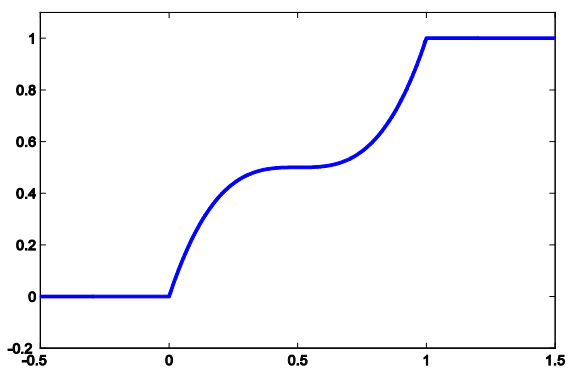

(a) $v(x)$

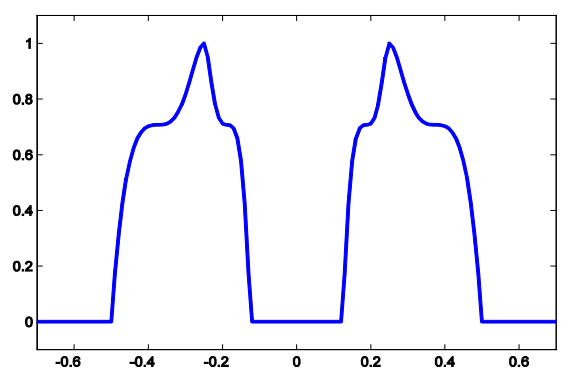

(b) $b(\omega)$

Fig 1: The two auxiliary functions $v$ and $b$

Up to now we have two auxiliary functions $v$ and $b$. In order to construct shearlets, we need another two functions $\psi_{1}$ and $\psi_{2}$. Now we define the function $\psi_{1}: \square \rightarrow \square \quad$ via its Fourier transform as

$$
\hat{\psi}_{1}(\omega)=\sqrt{b^{2}(2 \omega)+b^{2}(\omega)}
$$

Investigating the property of $\psi_{1}$, we have the following theorem. 


\section{Theorem 3.1}

The function $\hat{\psi}_{1}$ has $\operatorname{supp} \hat{\psi}_{1}=\left[-\frac{1}{2},-\frac{1}{16}\right] \bigcup\left[\frac{1}{16}, \frac{1}{2}\right]$ and fulfills

$$
\sum_{j \geq 0}\left|\hat{\psi}_{1}\left(2^{-2 j} \omega\right)\right|^{2}=1 \quad \text { for }|\omega| \geq \frac{1}{8}
$$

Since we can obtain the relation after elementary calculation, we omit the proof for this theorem. A plot of $\hat{\psi}_{1}$ is shown in Fig.2.

Recalling the definitions of $b$ and $\hat{\psi}_{1}$, we have

$$
\sum_{j \geq 0}\left|\hat{\psi}_{1}\left(2^{-2 j} \omega\right)\right|^{2}= \begin{cases}0 & \text { for }|\omega| \leq \frac{1}{16} \\ \sin ^{2}\left(\frac{\pi}{2} v(16|\omega|-1)\right) & \text { for } \frac{1}{16}<|\omega|<\frac{1}{8} \\ 1 & \text { for }|\omega| \geq \frac{1}{8}\end{cases}
$$

Next, we define the function $\psi_{2}: \square \rightarrow \square$ as

$$
\hat{\psi}_{2}(\omega)= \begin{cases}\sqrt{v(1+\omega)} & \omega \leq 0 \\ \sqrt{v(1-\omega)} & \omega>0\end{cases}
$$

In analogy with the above calculation, we have the following theorem.

\section{Theorem 3.2}

The function $\psi_{2}$ defined in (11) fulfills

$$
\sum_{l=-2^{j}}^{2^{j}}\left|\hat{\psi}_{2}\left(2^{j} \omega-l\right)\right|^{2}=1 \quad \text { for } j \geq 0,|\omega| \leq 1
$$

We also omit the concise proof for theorem 3.2. A plot of $\hat{\psi}_{2}$ is shown in Fig.2.
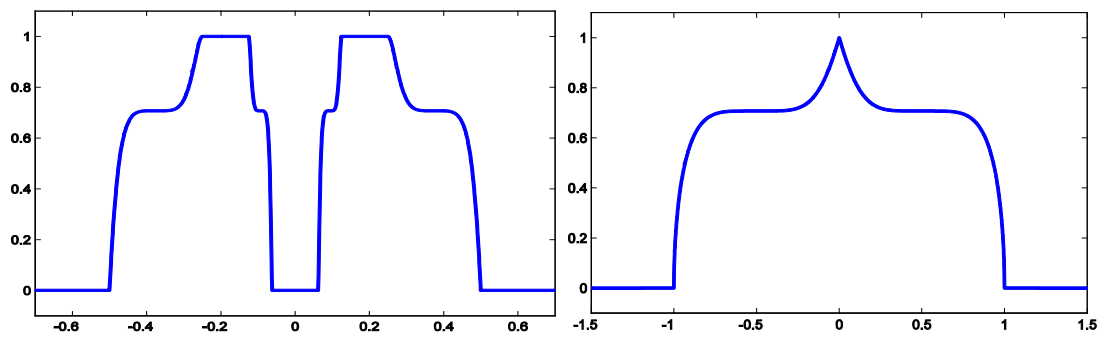

Fig. 2 The functions $\hat{\psi}_{1}$ and $\hat{\psi}_{2}$ 
Up to now we have obtained two important functions $\hat{\psi}_{1}$ and $\hat{\psi}_{2}$. For the construction of shearlets, we introduce the theory of affine systems with composite dilations[3]. In dimension $n=2$, the affine systems with composite dilations are the collections of the form:

$$
\left\{\psi_{j l k}(x)=|A|^{\frac{j}{2}} \psi\left(B(l) A^{j} x-k\right): j, l \in \square, k \in \square^{2}\right\}
$$

where $\psi \in L^{2}\left(\square^{2}\right)$, A, $B$ are $2 \times 2$ invertible matrices and $\operatorname{det}(B)=1$.

Now we introduce a theorem which provides a theoretical background for the construction of shearlets.

\section{Theorem 3.3}

Let $\Gamma=\left(-\frac{1}{2}, \frac{1}{2}\right], \quad A=\left(\begin{array}{ll}4 & 0 \\ 0 & 2\end{array}\right)$ and $B(l)=\left(\begin{array}{ll}1 & l \\ 0 & 1\end{array}\right)$, and suppose that

$$
\sum_{j \in Z}\left|\widehat{\psi_{1}}\left(4^{-j} x\right)\right|^{2}=1, \sum_{l \in Z}\left|\psi_{2}(x-l)\right|^{2}=1 \text { (a.e) }
$$

where $\widehat{\psi_{1}}, \psi_{2} \in L^{\infty}(R)=\left\{f\left|\|f\|_{\infty}=\operatorname{essup}_{R=(-\infty,+\infty)} f=\inf _{F_{0} \subset R, m F_{0}=0} \sup _{x \in R \mid F_{0}}\right| f(x) \mid<\infty\right\}$

For any $\omega=\left(\omega_{1}, \omega_{2}\right) \in \hat{\square}^{2}, \omega_{1} \neq 0$, let $\hat{\psi}$ be given by

$$
\hat{\psi}(\hat{\omega})=\psi\left(\omega_{1}, \omega_{2}\right)=\psi_{1}\left(\omega_{1}\right) \psi_{2}\left(\frac{\omega_{2}}{\omega_{1}}\right)
$$

If supp $\hat{\psi} \subset \Gamma^{2},\left\{\psi_{j l k}\right\}$ is a Parseval frame for $L^{2}\left(R^{2}\right)$, that is, for any $f \in L^{2}\left(R^{2}\right)$, we have

$$
\sum_{(j, l) \in Z^{2}} \sum_{k \in Z^{2}} k f, \psi_{j l k}>\left.\right|^{2}=\|f\|^{2}
$$

We further have

$$
f=\sum_{(j, l) \in Z^{2}} \sum_{k \in Z^{2}}<f, \psi_{j l k}>\psi_{j l k}
$$

where $\psi_{j l k}$ is defined as above.

Inspired by theorem 3.3, we consider shearlets from a different aspect. The following theorem allows us to construct shearlets on the cone. 


\section{Theorem 3.4}

Let $\Gamma=\left(-\frac{1}{2}, \frac{1}{2}\right], A_{1}=\left(\begin{array}{ll}4 & 0 \\ 0 & 2\end{array}\right), B_{1}(l)=\left(\begin{array}{ll}1 & l \\ 0 & 1\end{array}\right), A_{2}=\left(\begin{array}{ll}2 & 0 \\ 0 & 4\end{array}\right)$, $B_{2}(l)=\left(\begin{array}{ll}1 & 0 \\ l & 1\end{array}\right), C=A_{3}=B_{3}(l)=I$, $D_{1}=\left\{\omega=\left(\omega_{1}, \omega_{2}\right)\left|\frac{1}{8} \leq\right| \omega_{1}|,| \frac{\omega_{2}}{\omega_{1}} \mid \leq 1\right\}, D_{2}=\left\{\omega=\left(\omega_{1}, \omega_{2}\right)\left|\frac{1}{8} \leq\right| \omega_{2}|,| \frac{\omega_{1}}{\omega_{2}} \mid \leq 1\right\}$ $D_{3}=\left[-\frac{1}{8}, \frac{1}{8}\right]^{2} \quad \Delta_{1}=\Delta_{2}=\left\{(j, l) \mid-2^{j} \leq l \leq 2^{j}, j \in Z, j \geq 0\right\} \quad \Delta_{3}=\{(1,1)\}$ and suppose that

$$
\begin{gathered}
\sum_{j \geq 0}\left|\hat{\psi}_{1}\left(4^{-j} x\right)\right|^{2}=1 \text { for }|x| \geq \frac{1}{8} \\
\sum_{l=-2^{j}}^{2^{j}}\left|\hat{\psi}_{2}\left(2^{j} x-l\right)\right|^{2}=1 \text { for } j \geq 0,|x| \leq 1
\end{gathered}
$$

where $\hat{\psi_{1}}, \psi_{2} \in L^{\infty}(R)$.

$$
\begin{aligned}
& \text { For any } \omega=\left(\omega_{1}, \omega_{2}\right) \in \hat{\square}^{2}, \omega_{1} \neq 0 \text {, let } \hat{\psi}^{1}, \psi^{2} \text { and } \hat{\psi}^{3} \\
& \qquad \begin{aligned}
& \hat{\psi}^{1}(\omega)=\psi^{1}\left(\omega_{1}, \omega_{2}\right)=\psi_{1}\left(\omega_{1}\right) \psi_{2}\left(\frac{\omega_{2}}{\omega_{1}}\right) \\
& \hat{\psi}^{2 / 2}(\omega)=\psi\left(\omega_{1}, \omega_{2}\right)=\psi_{1}\left(\omega_{2}\right) \psi_{2}\left(\frac{\omega_{1}}{\omega_{2}}\right) \\
& \hat{\psi}^{3}(\omega)=\chi_{D_{3}}
\end{aligned}
\end{aligned}
$$

If supp $\hat{\psi}^{1}$, supp $\psi^{2} \subset \Gamma^{2}$, then $\left\{\psi^{\lambda}{ }_{j l k}\right\}_{(j, l) \in \Delta_{\lambda}, \lambda=1,2,3, k \in Z}$ is a Parseval frame for $L^{2}\left(R^{2}\right)$, where $\psi^{\lambda}{ }_{j l k}(x)=\left|A_{\lambda}\right|^{\frac{j}{2}} \psi^{\lambda}\left(B_{\lambda}(l) A^{j}{ }_{\lambda} x-k\right), \lambda=1,2,3$. Then, for any $f \in L^{2}\left(R^{2}\right)$, we have

$$
\begin{aligned}
& \sum_{j=0}^{\infty} \sum_{l=-2^{j}}^{2^{j}} \sum_{k=-\infty}^{\infty}\left|<f, \psi^{1}{ }_{i j k}>\right|^{2}+\sum_{j=0}^{\infty} \sum_{l=-2^{j}}^{2^{j}} \sum_{k=-\infty}^{\infty}\left|<f, \psi^{2}{ }_{i j k}>\right|^{2} \\
& +\sum_{j=0}^{\infty} \sum_{l=-2^{j}}^{2^{j}} \sum_{k=-\infty}^{\infty}\left|<f, \psi_{i j k}^{3}>\right|^{2}=\|f\|^{2}
\end{aligned}
$$

We further obtain 


$$
f=\sum_{j=0}^{\infty} \sum_{l=-2^{j}}^{2^{j}} \sum_{k=-\infty}^{\infty}\left|<f, \psi_{i j k}^{1}>\right|^{2}+\sum_{j=0}^{\infty} \sum_{l=-2^{j}}^{2^{j}} \sum_{k=-\infty}^{\infty}\left|<f, \psi^{2}{ }_{i j k}>\right|^{2}+\sum_{k=-\infty}^{\infty}\left|<f, \psi^{3}{ }_{k}>\right|^{2}
$$

Especially, we have

$$
\psi^{3}\left(x_{1}, x_{2}\right)=\frac{\sin \frac{\pi X_{1}}{4} \sin \frac{\pi X_{2}}{4}}{\pi^{2} X_{1} X_{2}}
$$

By the definition of $\widehat{\psi_{1}}, \psi_{2}$, one can easily observe that $\operatorname{supp} \hat{\psi}^{1}$, $\operatorname{supp} \psi^{2}$ $\subset\left(-\frac{1}{2}, \frac{1}{2}\right]^{2}$. Consequently, we have obtained one class of shearlets on the cone.

For the center part $D_{3}=\left[-\frac{1}{8}, \frac{1}{8}\right]^{2}$, we can define another set of functions $\hat{\psi}_{\mathrm{k}}^{3}$. The function $\psi^{3}$ can be defined as follows

$$
\hat{\psi}^{3}\left(\omega_{1}, \omega_{2}\right)= \begin{cases}\varphi\left(\omega_{1}\right) & \text { for }\left|\omega_{1}\right| \leq \frac{1}{8},\left|\omega_{2}\right| \leq\left|\omega_{1}\right| \\ \varphi\left(\omega_{2}\right) & \text { for }\left|\omega_{2}\right| \leq \frac{1}{8},\left|\omega_{1}\right| \leq\left|\omega_{2}\right|\end{cases}
$$

where

$$
\varphi(\omega)= \begin{cases}1 & \text { for }|\omega| \leq \frac{1}{16} \\ \cos \left(\frac{\pi}{2} v(16|\omega|-1)\right) & \text { for } \frac{1}{16}<|\omega|<\frac{1}{8} \\ 0 & \text { otherwise }\end{cases}
$$

Then we can obtain

$$
\hat{\phi}\left(\omega_{1}, \omega_{2}\right)= \begin{cases}1 & \text { for }\left|\omega_{1}\right| \leq \frac{1}{16},\left|\omega_{2}\right| \leq \frac{1}{16} \\ \cos \left(\frac{\pi}{2} v\left(16\left|\omega_{1}\right|-1\right)\right) & \text { for } \frac{1}{16}<\left|\omega_{1}\right|<\frac{1}{8},\left|\omega_{2}\right| \leq\left|\omega_{1}\right| \\ \cos \left(\frac{\pi}{2} v\left(16\left|\omega_{2}\right|-1\right)\right) & \text { for } \frac{1}{16}<\left|\omega_{2}\right|<\frac{1}{8},\left|\omega_{1}\right| \leq\left|\omega_{2}\right| \\ 0 & \text { otherwise }\end{cases}
$$

Analogously, we have the following theorem from which one can obtain a new class of shearlets on the cone. 


\section{Theorem 3.5}

Let

$$
\begin{aligned}
& C^{h}=\left\{\left(\omega_{1}, \omega_{2}\right) \in R^{2}:\left|\omega_{1}\right| \geq \frac{1}{8},\left|\omega_{1}\right|>\left|\omega_{2}\right|\right\}, \\
& C^{v}=\left\{\left(\omega_{1}, \omega_{2}\right) \in R^{2}:\left|\omega_{2}\right| \geq \frac{1}{8},\left|\omega_{2}\right|>\left|\omega_{1}\right|\right\}, \\
& C^{\times}=\left\{\left(\omega_{1}, \omega_{2}\right) \in R^{2}:\left|\omega_{1}\right| \geq \frac{1}{8},\left|\omega_{2}\right| \geq \frac{1}{8},\left|\omega_{2}\right|=\left|\omega_{1}\right|\right\} \\
& C^{0}=\left\{\left(\omega_{1}, \omega_{2}\right) \in R^{2}:\left|\omega_{1}\right|<\frac{1}{8},\left|\omega_{2}\right|<\frac{1}{8}\right\}, \\
& \Delta_{\lambda}^{\prime}=\left\{(j, l) \mid j \geq 0,-2^{j}+1 \leq l \leq 2^{j}-1\right\}, \lambda=1,2,
\end{aligned}
$$

and suppose that

$$
\begin{gathered}
\sum_{j \geq 0}\left|\hat{\psi}_{1}\left(4^{-j} x\right)\right|^{2}=1 \text { for }|x| \geq \frac{1}{8} \\
\sum_{l=-2^{j}}^{2^{j}}\left|\hat{\psi}_{2}\left(2^{j} x-l\right)\right|^{2}=1 \text { for } j \geq 0,|x| \leq 1
\end{gathered}
$$

where $\hat{\psi_{1}}, \psi_{2} \in L^{\infty}(R)$.

For any $\omega=\left(\omega_{1}, \omega_{2}\right) \in \hat{\square}^{2}, \omega_{1} \neq 0$, let $\hat{\psi}^{1}, \psi^{2}, \hat{\psi}^{3}$ and $\hat{\psi}^{\times}$be given by

$$
\begin{array}{cl}
\hat{\psi}^{\wedge}\left(\omega_{1}, \omega_{2}\right)=\psi_{1}\left(\omega_{1}\right) \psi_{2}\left(\frac{\omega_{2}}{\omega_{1}}\right), & \hat{\psi}^{2}\left(\omega_{1}, \omega_{2}\right)=\psi_{1}\left(\omega_{2}\right) \psi_{2}\left(\frac{\omega_{1}}{\omega_{2}}\right), \\
\psi^{3}{ }_{k}=\phi(x-k), & \hat{\psi}^{\times}\left(\omega_{1}, \omega_{2}\right)=\psi\left(\omega_{1}, \omega_{2}\right) \chi_{C^{\times}} .
\end{array}
$$

Then $\left\{\psi^{3}{ }_{k}: k \in \square^{2}\right\} \bigcup\left\{\psi^{\lambda}{ }_{j k}:(j, l) \in \Delta_{\lambda}, k \in \square^{2}, \lambda=1,2\right\} \bigcup\left\{\psi^{\times}{ }_{j k}: j \geq 0, l= \pm 2^{j}\right\}$

is a Parseval frame for $L^{2}\left(\square^{2}\right)$ and hence, for any $f \in L^{2}\left(R^{2}\right)$, we have

$$
f=\sum_{\lambda=1}^{2} \sum_{j=0}^{\infty} \sum_{l=-2^{j}+1}^{2^{j}-1} \sum_{k \in \mathbb{1}^{2}}<f, \psi^{\lambda}{ }_{j k}>\psi^{\lambda}{ }_{j k k}+\sum_{j=0}^{\infty} \sum_{l= \pm 2^{j}} \sum_{k \in \mathbb{I}^{2}}<f, \psi^{\times}{ }_{j k}>\psi_{j l k}^{\times}+\sum_{k \in \mathbb{I}^{2}}<f, \psi^{3}{ }_{k}>\psi^{3}{ }_{k}
$$




\section{Conclusions}

In this paper, we have succeeded in introducing the shearlet representation for the efficient geometric representation of multidimentional data. In particular, we present a method to construct shearlets in frequency domain. This method helped us to construct several types of shearlets in different aspects. First, a parseval frame of shearlets is obtained by combing different systems of cone-based shearlets. Subsequently, we construct a family of cone-adapted shearlet frame consisting of compactly supported shearlets. From mathematical perspective, one can consider smoothness of constructed shearlets. Especially, we can try to provide a new construction yielding smooth Parseval frame of shearlets. In future research, we intend to study some implementations of shearlet transform.

Acknowledgement Our project was supported by Zhejiang University of Technology.

\section{References}

1. Häuser, S. (2012). Fast Finite Shearlet Transform.

2. Guo, K., Kutyniok, G., \& Labate, D. (2006). Sparse multidimensional representations using anisotropic dilation and shear operators. Wavelets und Splines (Athens, GA, 2005), G. Chen und MJ Lai, eds., Nashboro Press, Nashville, TN, 189-201.

3. Easley, G., Labate, D., \& Lim, W. Q. (2008). Sparse directional image representations using the discrete shearlet transform. Applied and Computational Harmonic Analysis, 25(1), 25-46.

4. Guo, K., \& Labate, D. (2013). The construction of smooth parseval frames of shearlets. Mathematical Modelling of Natural Phenomena, 8(01), 82-105.

5. Guo, K., \& Labate, D. (2007). Optimally sparse multidimensional representation using shearlets. SIAM journal on mathematical analysis,39(1), 298-318.

6. Lim, W. Q. (2010). The discrete shearlet transform: A new directional transform and compactly supported shearlet frames. Image Processing, IEEE Transactions on, 19(5), 1166-1180.

7. Kutyniok, G., Lim, W. Q., \& Zhuang, X. (2012). Digital Shearlet Transforms. In Shearlets (pp. 239-282). Birkhäuser Boston.

8. Candes, E., Demanet, L., Donoho, D., \& Ying, L. (2006). Fast discrete curvelet transforms. Multiscale Modeling \& Simulation, 5(3), 861-899.

9. Candès, E. J., \& Donoho, D. L. (1999). Ridgelets: A key to higher-dimensional intermittency?. Philosophical Transactions of the Royal Society of London. Series A Mathematical, Physical and Engineering Sciences,357(1760), 2495-2509.

10. Do, M. N., \& Vetterli, M. (2005). The contourlet transform: an efficient directional multiresolution image representation. Image Processing, IEEE Transactions on, 14(12), 2091-2106.

11. Candes, E. J., \& Donoho, D. L. (2004). New tight frames of curvelets and optimal representations of objects with piecewise C2 singularities.Communications on pure and applied mathematics, 572), 219-266. 